Service social

\title{
Milieux sociaux d'appartenance et représentations de la santé chez un groupe de femmes âgées francophones du Nouveau-Brunswick
}

\section{Ysabel Provencher}

Volume 50, numéro 1, 2003

URI : https://id.erudit.org/iderudit/008507ar

DOI : https://doi.org/10.7202/008507ar

Aller au sommaire du numéro

Éditeur(s)

École de service social de l'Université Laval

ISSN

1708-1734 (numérique)

Découvrir la revue

Citer cet article

Provencher, Y. (2003). Milieux sociaux d'appartenance et représentations de la santé chez un groupe de femmes âgées francophones du Nouveau-Brunswick. Service social, 50(1), 231-262. https://doi.org/10.7202/008507ar
Résumé de l'article

Le présent article fait état des résultats d'une étude portant sur l'influence qu'exerce le milieu social d'appartenance sur les croyances, les connaissances et les comportements associés à la santé et à la maladie chez un groupe de femmes âgées francophones demeurant dans la région urbaine de Moncton, au Nouveau-Brunswick. L'objectif de cette recherche consistait à repérer des représentations de la santé chez trois groupes de femmes âgées issues du milieu populaire, de la classe moyenne et de l'élite locale, introduisant ainsi la notion de culture de milieu et son incidence sur les attitudes et les comportements associés à la santé. Les résultats de cette étude nous ont permis de dégager un certain nombre d'idées partagées par l'ensemble des répondantes au sujet de la santé et de la maladie ainsi que de mettre en lumière des éléments de représentation de la santé propres aux femmes issues du milieu populaire, d'une part, et de la classe moyenne, d'autre part. Les résultats obtenus ne nous ont cependant pas permis de dégager des éléments constitutifs d'une représentation de la santé qui soit caractéristique des femmes faisant partie de l'élite. Globalement, pour les femmes âgées issues du milieu populaire, la santé et la maladie s'expliquent en référence au destin, à la chance/malchance, au travail ainsi qu'aux habitudes de vie. Pour les femmes âgées issues de la classe moyenne, la santé et la maladie sont davantage liées à la responsabilité et à la volonté personnelles. Quant au discours des femmes issues du milieu de l'élite sur la santé et la maladie, il est caractérisé davantage par le thème du contraste entre le monde passé et le monde actuel. Cette étude contribue directement à l'élargissement des connaissances sur la diversité relative aux milieux d'appartenance dans le champ de la gérontologie sociale et sur l'impact qu'elle a sur l'intervention sociale et sanitaire.
Ce document est protégé par la loi sur le droit d'auteur. L'utilisation des services d’Érudit (y compris la reproduction) est assujettie à sa politique d'utilisation que vous pouvez consulter en ligne.

https://apropos.erudit.org/fr/usagers/politique-dutilisation/ 


\section{Milieux sociaux d'appartenance et représentations de la santé chez un groupe de femmes âgées francophones du Nouveau-Brunswick}

Ysabel PROVENCHER

Professeure adjointe Université de Moncton Courriel : proveny@umoncton.ca

Le présent article fait état des résultats d'une étude portant sur l'influence qu'exerce le milieu social d'appartenance sur les croyances, les connaissances et les comportements associés à la santé et à la maladie chez un groupe de femmes âgées francophones demeurant dans la région urbaine de Moncton, au Nouveau-Brunswick. L'objectif de cette recherche consistait à repérer des représentations de la santé chez trois groupes de femmes âgées issues du milieu populaire, de la classe moyenne et de l'élite locale, introduisant ainsi la notion de culture de milieu et son incidence sur les attitudes et les comportements associés à la santé. Les résultats de cette étude nous ont permis de dégager un certain nombre d'idées partagées par l'ensemble des répondantes au sujet de la santé et de la maladie ainsi que de mettre en lumière des éléments de représentation de la santé propres aux femmes issues du milieu populaire, d'une part, et de la classe moyenne, d'autre part. Les résultats obtenus ne nous ont cependant pas permis de dégager des éléments constitutifs d'une représentation de la santé qui soit caractéristique des femmes faisant partie de l'élite. Globalement, pour les femmes âgées issues du milieu populaire, la santé et la maladie s'expliquent en référence au destin, à la chance/malchance, au travail ainsi qu'aux habitudes de vie. Pour les femmes âgées issues de la classe 
moyenne, la santé et la maladie sont davantage liées à la responsabilité et à la volonté personnelles. Quant au discours des femmes issues du milieu de l'élite sur la santé et la maladie, il est caractérisé davantage par le thème du contraste entre le monde passé et le monde actuel. Cette étude contribue directement à l'élargissement des connaissances sur la diversité relative aux milieux d'appartenance dans le champ de la gérontologie sociale et sur l'impact qu'elle a sur l'intervention sociale et sanitaire.

Mots clés : comportements associés à la santé, croyances associées à la santé, femmes âgées, représentation de la santé, utilisation des services.

This article presents the results of a study on the influence of social environment on the beliefs, knowledge and behaviours related to health and illness in a group of elderly French-speaking women living in the urban area of Moncton, New Brunswick. The purpose of the study was to identify social representations of health by three groups of women belonging to the working class, the middle class and the local elite, and thus to introduce the concept of class culture and its incidence on health-related attitudes and behaviours. Based on the results of the study, a certain number of ideas were identified that were shared by all informants concerning health and illness, and some elements of a social representation of health emerged that were specific to working class and to middle class women, respectively. The results did not enable identification of constituents of a social representation of health that were specific to women from the elite. Overall, for elderly women from the working class, health and illness are explained in terms of fate, of good or bad luck, of work and of lifestyle. For elderly women from the middle class, explanations of health and illness are more closely related to personal will and responsibility. As for the women from the elite, their views were more focused on the contrast between the world as it was in the past and the world as it is currently. This study offers a direct contribution to a better understanding of diversity due to class membership in the field of social gerontology and of its impact on social intervention and health care.

Key words : health behaviours, health beliefs, health beliefs, elderly women, social representation of health, health care use. 


\section{INTRODUCTION}

Centrée sur l'influence qu'exerce le milieu d'appartenance sur les représentations de la santé chez les femmes âgées, la recherche ${ }^{1}$ dont nous rendons compte dans cet article s'inscrit à l'intérieur du champ de réflexion sur les rapports entre vieillesse, culture et santé (Provencher et Lepage, 2003). Globalement, dans notre étude, nous avons cherché à comparer des éléments de représentation de la santé chez des femmes âgées francophones de la région de Moncton, au Nouveau-Brunswick, en fonction de leur milieu ou de leur classe sociale d'appartenance. Le présent article rend compte tout d'abord de l'état de la recherche sur le rapport entre personnes âgées et santé, puis présente les principaux concepts utilisés dans notre étude. La méthode employée est expliquée en troisième partie de cet article, tandis que nous traitons dans la dernière section des principaux résultats de l'analyse comparative des représentations de la santé et de la maladie chez les femmes rencontrées. Enfin, conformément à d'autres travaux menés sur les représentations sociales de la santé, les résultats obtenus dans notre recherche mettent en lumière différentes manières de connaître, de penser et d'agir des personnes face au processus de maintien de leur santé, lesquelles varient selon les milieux d'appartenance. Nous soulignons plus particulièrement en conclusion de cet article le rôle important du service social dans le champ de la santé pour favoriser une meilleure compréhension des logiques sous-jacentes aux modes de gestion de la santé et de la maladie de différents groupes sociaux, et, de ce fait, favoriser l'émergence d'une pratique sociosanitaire qui puisse mieux rendre compte de cette diversité.

\section{RECENSION DES ÉCRITS}

De manière générale, les recherches menées sur le thème de la santé des personnes âgées s'inscrivent dans une compréhension de la santé comme résultante de l'influence de plusieurs facteurs. Outre l'effort d'acquisition de connaissances sur l'état de santé des personnes âgées, on tente de saisir les relations entre un ou plusieurs déterminants psychologiques, biologiques, sociaux,

1. Cette recherche a été financée par la Drummond Foundation. 
culturels, de même que leur influence respective sur le rapport que les personnes âgées entretiennent avec la santé.

Une partie de ces recherches s'articule donc autour des facteurs associés à l'utilisation des services de santé. On y indique tour à tour que l'âge, le sexe, le niveau de scolarité, l'appartenance à un milieu rural ou urbain, l'origine ethnique, le revenu, la connaissance, la perception ou la disponibilité des services, l'état de santé ou le réseau informel peuvent influencer l'utilisation des services sociosanitaires par les personnes âgées (Krout, 1983; Corin, Tremblay, Sherif et Bergeron, 1984; Lubben, Weiler et Chi, 1989; Gerritsen, Wolffensperger et Van den Heuvel 1990; Leigh et Fries,1993; Sévigny et Vézina, 1996; Mitchell et Krout 1998). Pour certains auteurs, toutefois, les recherches portant sur l'utilisation de services font voir que les personnes âgées inventent leur propre modèle d'utilisation des services en fonction de leurs valeurs et que ces valeurs sont modelées par leur trajectoire de vie, d'où l'importance de considérer, en amont de l'agir, les perceptions, croyances et attitudes qui influencent les conduites relatives à la santé et à la maladie chez les personnes âgées. À cet égard, les recherches portant sur les valeurs, les croyances et les connaissances associées à la santé, considérées comme autant de facteurs prédisposant à l'utilisation des services ou, plus largement, à l'adoption de comportements liés à la santé, ont permis de mettre en lumière les relations entre certaines caractéristiques relatives aux individus et les significations que ceux-ci donnent à la santé (Moen, 1978; Krout, 1983; Calnan, 1986; Ankri et Henrard, 1994; Schulze et Welters, 1994; Ferrini, Edelstein et Barret-Connor, 1994; Arcury, Quandt et Bell, 2001; Provencher, 2002). Ces recherches mettent en lumière le fait que ces valeurs, croyances et connaissances sont en partie déterminées par les milieux sociaux dans lesquels vivent les personnes, rendant ainsi nécessaire un approfondissement des liens entre milieu social d'appartenance des individus et sens donnés à la santé chez les personnes âgées. Dans cette perspective, l'étude de Calnan (1986) sur les perceptions des femmes âgées face au maintien de la santé illustre bien l'influence de la classe sociale d'appartenance sur les connaissances des femmes âgées relativement aux conduites de santé. Fondamentalement, les résultats de cette étude montrent que le raisonnement des femmes issues des classes populaires 
(working class) au sujet de la santé et de la maladie est articulé en fonction des connaissances issues de l'expérience, tandis que le raisonnement des femmes issues de la classe moyenne est davantage articulé autour d'une conception multidimensionnelle de la maladie, ce qui se retrouve également dans les résultats de notre étude. En ce qui a trait à la prévention de la maladie, l'étude de Calnan révèle que les femmes issues des classes moyennes et supérieures sont beaucoup plus nombreuses que celles issues des classes populaires à considérer la possibilité de prévenir l'apparition des maladies. L'auteur conclut sur le fait que les croyances et les savoirs associés à la santé représentent des attributs des environnements sociaux, économiques et culturels dans lesquels vivent les personnes. Dix ans plus tard, faisant écho à ces considérations, S.J. Williams (1995) déplore le peu de connaissances que nous avons de cette influence qu'exerce le milieu social sur les comportements et les croyances associés à la santé. Selon lui, en dépit d'une vaste production de connaissances empiriques au cours des vingt dernières années, relativement peu d'efforts ont été faits pour théoriser davantage sur les relations entre l'appartenance à un milieu social donné, les styles de vie et leurs correspondances avec les comportements associés à la santé. Williams fait valoir que l'appartenance à un milieu social détermine les croyances et les connaissances en matière de santé, lesquelles exercent à leur tour une influence déterminante sur les comportements des personnes en matière de santé.

Ces résultats, de même que ceux évoqués précédemment sur les comportements, les valeurs, les croyances et les savoirs relatifs à la santé et à la maladie dans différents groupes sociaux montrent l'importance d'approfondir notre connaissance et notre compréhension du sens donné à la santé par les personnes âgées en fonction de leur milieu d'appartenance. Dans la démarche de recherche dont nous rapportons les résultats, nous avons voulu en fait répondre à la question suivante: Quelles sont les représentations sociales de la santé que partagent les femmes âgées issues d'un même milieu social? II s'agissait d'un pas de plus dans la vérification des liens entre milieu d'appartenance et représentation de la santé. En adoptant une structure de preuve articulée en fonction d'un devis de recherche qualitatif, le défi consistait à repérer les dénominateurs communs des images mentales qui 
conditionnent la pratique de groupes d'individus, tout en rendant opérationnelle la prise en compte des milieux d'appartenance qui fondent la composition de ces groupes. Les concepts de représentation sociale de la santé et de milieu-classe sociale ont ainsi constitué les concepts principaux de notre étude et leur décomposition en indicateurs des voies d'accès a été nécessaire pour analyser les liens possibles entre représentations et appartenances sociales.

\section{CADRE de RÉFÉRENCE}

\section{Le concept de représentation sociale de la santé}

Sorte de système de pensée commun à plusieurs, la représentation sociale rassemble un ensemble cohérent de savoirs, d'opinions, de croyances et d'attitudes et elle donne un sens à l'action d'un groupe social (Séca, 2001). Par conséquent, si le rapport à la santé des individus est marqué par les contextes sociaux et culturels dans lesquels il se déploie, la recherche de connaissances sur ces objets culturels que sont les représentations de la santé devient une voie privilégiée pour saisir les logiques d'ensemble qui sous-tendent les attitudes, les croyances et les comportements relatifs à la santé et à la maladie au sein de différents groupes sociaux. Autrement dit, le concept de représentation sociale appliqué à la santé permet de comprendre davantage l'influence des dimensions sociales et culturelles sur les pensées et les actions des individus au regard de leur processus à la fois de maintien de la santé et de gestion de la maladie (Jodelet, 1992; Joffe, 1999). De manière générale, les études sur les représentations de la santé comportent divers éléments reliés aux croyances, aux connaissances et aux modes de gestion de la maladie, cette dernière étant considérée comme étant à l'opposé de la santé et servant à enrichir la connaissance au sujet des représentations de la santé communes à différents groupes sociaux. Ainsi, un certain nombre d'études se sont attardées à rendre compte des éléments de représentation de la santé propres aux personnes issues de milieux défavorisés (De Koninck et Guyon, 1985; Paquet, 1989; Colin, Ouellet, Boyer et Martin, 1992; Godin, 1993; Provencher, 2002). Nous appuyant sur ces travaux, nous 
avons établi des indicateurs permettant de recueillir de l'information sur les origines, les définitions et les conduites associées à la santé et à la maladie.

\section{Du statut socioéconomique au milieu d'appartenance}

Une partie des études évoquées précédemment sur la relation que les personnes âgées établissent avec la santé mettent en lumière le rapport étroit entre les processus de maintien de la santé et le statut socioéconomique des individus, celui-ci étant le plus souvent articulé en fonction des indicateurs de niveau de scolarité, de type d'emploi occupé, de revenu personnel ou de revenu du ménage, ces indicateurs pouvant être considérés isolément ou reliés entre eux. En fait, si le «statut socioéconomique » est évoqué comme un facteur déterminant de comportements associés à la santé, les indicateurs servant à opérationnaliser cette notion varient selon les recherches, rendant plus opaque la compréhension de l'incidence du statut socioéconomique sur ces comportements (Roos et Mustard, 1997; Provencher, 1999; Grzywacz, 2000). Nous avons donc cherché à délimiter l'objet de recherche à l'intérieur d'une grille des milieux-classes sociales comprenant le milieu populaire, le milieu de la classe moyenne et l'élite, que nos voyons fondamentalement comme des effets de la structure des rapports sociaux. Les classes sociales ainsi définies renvoient en fait à des positions différentes au sein de la structure sociale. Pour les besoins de notre étude, nous avons retenu la typologie des classes sociales élaborée par Gérald Doré, principalement pour la composition des indicateurs économiques, sociaux et culturels qu'elle sous-tend (Doré, 1988). Cette typologie, déjà utilisée lors de recherches antérieures (Bariteau, 1985; Doré, 1991; Provencher, 1999; Provencher, 2002), renvoie à des positions déterminées dans le processus de production-reproduction sociale en fonction de deux indicateurs, soit le degré de contrôle exercé dans les activités de production-reproduction sociale et la fonction politique ou idéologique exercée ou non dans le processus de division du travail à l'intérieur du système de production capitaliste. Fondamentalement, la présence ou l'absence des attributs repérés permet de définir l'appartenance des individus aux divers milieux sociaux. Ainsi, le milieu populaire est associé à une absence de contrôle sur les activités de production-reproduction de même 
qu'à une fonction d'exécutant dans le mode de production. De plus, une fonction idéologique ou politique, même mineure, ainsi qu'un certain contrôle sur ses activités de production caractérisent l'appartenance au milieu de la classe moyenne, également appelée petite-bourgeoisie salariée. Le milieu de l'élite, quant à lui, est associé à l'exercice d'une fonction idéologique ou politique ainsi qu'à un contrôle important sur ses activités de production. Enfin, puisque la position occupée dans les rapports de production détermine globalement les manières d'être des individus, nous considérons, à l'instar de nombreux auteurs, que le concept de milieu social doit chercher à inclure des indicateurs qui traduisent plus directement une culture ou une manière de vivre propre aux gens qui le composent. Par conséquent, nous avons inclus l'indicateur du niveau de scolarité atteint dans la détermination de l'appartenance de milieu social. Essentiellement, un niveau de scolarité postsecondaire est propre aux personnes appartenant aux milieux de la classe moyenne et de l'élite (Paquet, 1989; Lalive D'Épinay, 1991).

\section{MÉTHODOLOGIE}

\section{Population à l'étude, mode de recrutement et considérations éthiques}

Dans notre recherche nous avons cherché à réduire le plus possible l'éventail des différences pouvant être associées à d'autres variables comme le sexe ou l'appartenance à un milieu urbain ou rural. Nous avons donc centré notre étude auprès de femmes en situation de retraite ${ }^{2}$, vivant à domicile, demeurant dans la région urbaine du grand Moncton et appartenant au milieu populaire, à celui de la classe moyenne ou à celui de l'élite locale. Par ailleurs, dans la mesure où les femmes rencontrées dans cette étude ne

2. Nous nous sommes appuyée sur la notion de retraite telle que définie par Statistique Canada où une personne âgée de 55 ans et plus est considérée comme retraitée si elle reçoit $50 \%$ ou plus de son revenu total de sources associées à la retraite. Les sources associées à la retraite comprennent celles provenant du Régime de pensions du Canada, du régime de Sécurité de la vieillesse, du régime du Supplément de revenu garanti, du Régime de rentes du Québec, des investissements et dividendes ainsi que des divers régimes de pensions de retraite et rentes (Statistique Canada, 2003). 
sont plus engagées dans le processus de production-reproduction sociale comme elles l'étaient au moment de leur vie dite active, nous considérons que leur appartenance de milieu demeure la même que durant leur âge dit actif. La majorité des participantes ont été sollicitées au moyen de rencontres d'information tenues au sein de diverses associations pour personnes âgées ou par l'entremise d'informateurs clés en contact avec les personnes âgées (secrétaires de paroisses, prêtres, animateurs et animatrices œuvrant dans le domaine du loisir, personnel des médias francophones, etc.). Au total, 39 entretiens individuels non directifs d'environ une heure chacun ont été réalisés. Les entrevues ont été enregistrées et menées par un assistant de recherche. Toutefois, du fait que la majorité des répondantes étaient ou avaient été mariées, nous avons cherché à retenir les situations d'homogénéité de classe sociale d'appartenance entre la femme et son conjoint en écartant les situations où l'appartenance de classe du conjoint différait de celle de la participante. Ainsi, 29 de ces entretiens ont servi à l'analyse comparative des représentations de la santé/ maladie dont il est question dans cet article. L'échantillon a été réparti en trois catégories de répondantes issues des trois milieux sociaux considérés dans cette étude, soit dix participantes issues du milieu populaire, dix de la classe moyenne et neuf participantes issues de l'élite ${ }^{3}$. Le tableau 1 présente la description des emplois occupés par les participantes. Dans le cas des femmes ayant œuvré surtout ou en totalité au foyer, nous nous sommes appuyée sur la position de classe du conjoint pour déterminer la position de classe de la participante. Cette manière de procéder, même si elle entraîne des limites évidentes au regard de l'analyse de ce qui fonde l'appartenance de milieu ou de classe d'une partie importante des femmes rencontrées dans cette étude, a toutefois été utilisée ou jugée adéquate par d'autres auteurs relativement à la prise en compte de l'appartenance de classe des femmes qui ne sont pas engagées d'égale façon dans les rapports de production (Calnan, 1986; MacFarlane, 1980; Dale, Gilbert et Arber,

3. L'ascension de la population acadienne à la classe de l'élite étant un phénomène relativement récent et la taille de la population à l'étude étant d'autant réduite, seules neuf femmes ont pu être recrutées pour la composition de ce groupe de l'échantillon, atteignant presque le même nombre que les deux autres groupes (voir : Daigle, 1993; Allain, 1998). 
1985). Enfin, de manière à établir un critère de validité externe dans notre concept de milieu-classe sociale, nous avons mis en parallèle la correspondance entre les emplois occupés dans chaque milieu ou chaque classe sociale et la Classification internationale type des professions (CITP-88) de l'Organisation internationale du travail (O.I.T.) (2003). 


\begin{tabular}{|c|c|c|c|}
\hline $\begin{array}{l}\text { Milieu- } \\
\text { classe } \\
\text { sociale }\end{array}$ & & Nature de l'emploi & $\begin{array}{c}\text { Correspondance au } \\
\text { CITP-88 }\end{array}$ \\
\hline \multirow{9}{*}{$\begin{array}{l}\text { Populaire } \\
\quad(10)\end{array}$} & 1 & Commis de magasin & \multirow{2}{*}{$\begin{array}{l}\text { Personnels des services et ven } \\
\text { deurs de magasin et de marché } \\
\text { (G.G. 5) }\end{array}$} \\
\hline & 2 & Serveuse de restaurant & \\
\hline & 1 & Caissière & \multirow{2}{*}{$\begin{array}{l}\text { Employés de type administratif } \\
\text { (G.G. 4) }\end{array}$} \\
\hline & 1 & Secrétaire & \\
\hline & (1) & Conducteur de train & $\begin{array}{l}\text { Conducteurs d'installation } \\
\text { et de machines et ouvriers } \\
\text { de l'assemblage (G.G. 8) }\end{array}$ \\
\hline & (1) & Électricien & $\begin{array}{l}\text { Artisans et ouvriers des métiers } \\
\text { de type artisanal (G.G. } 7 \text { ) }\end{array}$ \\
\hline & 1 & Ouvrière d'usine & \multirow{3}{*}{$\begin{array}{l}\text { Ouvriers et employés non } \\
\text { qualifiés (G.G. 9) }\end{array}$} \\
\hline & (1) & Bûcheron & \\
\hline & (1) & Commis voyageur & \\
\hline \multirow{4}{*}{$\begin{array}{l}\text { Moyenne } \\
\quad(10)\end{array}$} & 7 & Enseignante & $\begin{array}{l}\text { Professions intellectuelles } \\
\text { et scientifiques (G.G. 2) }\end{array}$ \\
\hline & 1 & Infirmière & \multirow{2}{*}{$\begin{array}{l}\text { Professions intermédiaires } \\
\text { (G.G. 3) }\end{array}$} \\
\hline & 1 & Technicienne & \\
\hline & 1 & Gérante & $\begin{array}{l}\text { Membres de l'exécutif } \\
\text { et des corps législatifs (G.G. } 1 \text { ) } \\
\text { Sous-groupe } 13: \text { dirigeants } \\
\text { et gérants }\end{array}$ \\
\hline \multirow{5}{*}{$\begin{array}{l}\text { Élite } \\
(9)\end{array}$} & (1) & Médecin & $\begin{array}{l}\text { Professions intellectuelles } \\
\text { et scientifiques (G.G. 2) }\end{array}$ \\
\hline & $\begin{array}{l}1+ \\
(2)\end{array}$ & $\begin{array}{l}\text { Cadre supérieur de l'ad- } \\
\text { ministration publique }\end{array}$ & \multirow{4}{*}{$\begin{array}{l}\text { Membres de l'exécutif } \\
\text { et des corps législatifs (G.G. 1) }\end{array}$} \\
\hline & $(2)$ & Propriétaire d'entreprise & \\
\hline & (2) & Politicien & \\
\hline & $(1)$ & Dirigeant de société & \\
\hline Total & 29 & & \\
\hline
\end{tabular}

4. La classification internationale inclut l'ensemble des cadres et des dirigeants dans le grand groupe 1. Or, la typologie que nous utilisons distingue un degré de contrôle différent entre la fonction de cadre intermédiaire et celle de cadre supérieur ou dirigeant, associant la première à la classe moyenne et la seconde à la classe de l'élite. 
Les données inscrites au tableau 1 permettent de constater une nette correspondance entre la typologie que nous avons utilisée et celle de l'Organisation internationale du travail, les trois milieuxclasses sociales correspondant à des ensembles de grands groupes de professions de la classification internationale, mis à part le cas des médecins que, à la différence de l'O.I.T., nous avons associés à la classe de l'élite, principalement à cause du degré de contrôle qu'ils exercent sur leurs activités professionnelles (Provencher, 2001). Enfin, en plus des caractéristiques liées au type d'emploi occupé, nos indicateurs de milieu-classe sociale tenaient compte du niveau de scolarité atteint. Ainsi, $90 \%$ des répondantes de milieux populaires ont un niveau de scolarité primaire ou secondaire, tandis que $100 \%$ des répondantes issues de la classe moyenne ont un niveau de scolarité postsecondaire. Les niveaux de scolarité atteints par les femmes issues de l'élite se répartissent pour leur part plus largement. Quant aux niveaux de scolarité atteints par les conjoints des participantes, ils sont analogues à ceux atteints par les participantes, ce qui confirme l'homogénéité de milieu que nous recherchions pour les participantes mariées ou veuves. Enfin, nous avons précisé nos considérations relativement aux questions éthiques soulevées par l'étude avant de rencontrer les personnes.

\section{Mode de collecte, instrument utilisé et plan d'analyse des données}

Ainsi que nous l'avons mentionné auparavant, nos données de recherche ont été recueillies au moyen d'entrevues menées auprès de 39 femmes aînées, dont 29 ont fait l'objet d'une étude plus approfondie. Dès lors, considérant les objectifs poursuivis dans notre étude, nous avons opté pour des entretiens non directifs particulièrement indiqués pour étudier les valeurs, les normes et les manières de penser propres à une culture donnée (Daunais, 1992). Toutefois, même s'il s'agissait d'entretiens non directifs, nous avions préparé un guide, qui allait servir d'outil au besoin pour favoriser l'expression des participantes à propos des origines, des définitions et des conduites qu'elles associent à la santé et à la maladie. Les principales questions du guide étaient les suivantes: Pour vous, si on vous demandait c'est quoi la santé, que diriez-vous? Selon vous, qu'est-ce qui fait qu'une personne 
est en santé? Qu'est-ce qu'il faut faire pour rester en santé? Qu'est-ce qui fait qu'une personne devient malade? La maladie, ça dépend de quoi? Dans le fond, la maladie, selon vous, ça se définit comment? Qu'est-ce qu'il faut faire lorsqu'on est malade pour redevenir en santé? Le logiciel de données textuelles NVivo a servi d'outil principal pour les opérations de traitement et d'analyse des données. Enfin, considérant que le vocabulaire, le recours à des notions abstraites ou à l'expérience concrète pour expliquer une idée et le degré d'aisance manifeste à discourir avec un intervieweur pouvaient varier fortement selon l'appartenance de classe des répondantes, nous avons centré l'analyse sur le repérage de la présence ou absence des idées relatives aux origines, aux définitions et aux conduites de santé et de maladie et non pas sur la fréquence d'apparition des unités à l'intérieur d'un discours. Autrement dit, chaque idée relative à l'un ou l'autre aspect des représentations de la santé a été prise en compte dans l'analyse que nous avons faite de chacun des discours, sans égard à l'ampleur que pouvait prendre cette idée par rapport aux autres idées exprimées dans un même entretien, ce qui représente par ailleurs une limite importante de notre étude.

\section{Limites de l'étude}

Au-delà des limites déjà évoquées concernant la détermination de l'appartenance de classe des répondantes par la référence aux conjoints et la mesure de l'importance des idées les unes par rapport aux autres dans l'analyse que nous avons faite des entretiens, d'autres limites méritent d'être soulignées. L'une d'entre elles est liée à la stratégie de recrutement des participantes, qui a conduit à une prépondérance de membres actives dans les associations volontaires de personnes âgées parmi les répondantes. En effet, mis à part le cas des femmes de l'élite, où nous avons procédé davantage par l'intermédiaire d'informateurs clés, la très grande majorité des femmes rencontrées ont été recrutées à partir d'associations d'aînés. Or, les travaux de Delisle et Ouellet (2002) démontrent l'influence de la participation sociale sur l'utilisation de services de santé et, plus largement, sur les comportements associés à la santé et à la prévention de la maladie. Cela explique les résultats obtenus dans notre étude concernant la pratique de 
l'activité physique comme conduite de santé, que nous verrons dans la partie suivante.

\section{RÉSULTATS}

Les résultats rapportés dans cette section rendent compte des représentations sociales de la santé des femmes issues des trois milieux sociaux considérés dans notre étude. Bien que les résultats ne puissent être généralisables à l'ensemble de la population à l'étude, ils revêtent un intérêt certain au regard de la connaissance qu'ils apportent quant aux différences et aux ressemblances constatées dans les discours sur la santé et la maladie en fonction des milieux étudiés. Ces résultats révèlent que les femmes rencontrées partagent un certain nombre d'idées à propos de la santé et de la maladie, mais les logiques d'articulation de ces idées varient. Les résultats obtenus montrent également des différences dans le discours des unes et des autres selon le milieu ou la classe sociale d'appartenance.

\section{Des idées partagées sur la santé et la maladie}

Comme nous pouvons le constater dans le tableau 2, certaines idées au sujet de la santé sont assez répandues chez l'ensemble des répondantes ayant participé à cette étude. Ainsi, pour 17 des 29 femmes interrogées, le travail ou l'occupation se trouve à l'origine de la santé. Travailler, ça fait pas mourir. L'idée de l'occupation évoquée ici tient à la fois de l'activité manuelle concrète (jardinage, entretien ménager...) et de celle faisant davantage appel aux capacités cognitives (mots cachés, lecture...), le premier type d'activité étant plus fréquemment rapporté par les femmes issues du milieu populaire. Les femmes appartenant aux deux autres milieux ont quant à elles établi un lien entre la santé et le fait de trouver, dans l'activité, un sens à l'existence: Tu sens que tu accomplis des choses. Ainsi, pour la majorité des participantes à notre étude, le travail - ou plus largement l'occupation - contribue à la santé, l'explication du lien entre les deux faisant toutefois appel à des logiques différentes. De même, une majorité des participantes à notre étude ont décrit leur perception de la santé comme résultant de facteurs extérieurs à la volonté individuelle. À l'intérieur de ce thème, l'idée du bagage héréditaire comme une 
réserve de santé héritée à la naissance a été évoquée dans des proportions similaires dans chacun des milieux, chez un total de 13 participantes. De même, un nombre presque identique de participantes de chaque milieu ont défini la santé de manière instrumentale, les capacités fonctionnelles servant de baromètre pour évaluer l'état de santé, soit un total de 14 femmes sur les 29 participantes.

Enfin, et il s'agit là d'un résultat imprévu dans notre étude, dans 27 des 29 entretiens analysés, les femmes ont fait référence à l'activité physique comme moyen pour conserver sa santé, indiquant clairement un lien entre le maintien de leur état de santé et l'activité du corps. Toutefois, si la quasi-totalité des répondantes ont dit reconnaître un lien entre le maintien de leur santé et l'activité physique, elles l'expriment de différentes façons. Pour les femmes issues de l'élite, l'activité physique est évoquée principalement comme un moyen d'améliorer son moral. Les répondantes de la classe populaire, pour leur part, ont vanté les bienfaits de l'activité sur la santé physique (sommeil plus réparateur, diminution de la sensation de fatigue). À la différence du discours des deux autres groupes, celui des femmes de la classe moyenne sur l'activité physique est nettement dominé par l'évaluation qu'elles font de leur propre pratique, distinguant nettement la part associée aux activités de la vie domestique de celle relative à l'amélioration de la condition physique.

\section{Tableau 2}

Les idées communes au sujet de la santé parmi les répondantes des différentes classes sociales

\begin{tabular}{|l|l|c|c|c|}
\hline \multicolumn{2}{|c|}{ Catégorie } & Populaire & Moyenne & Élite \\
\hline \multirow{2}{*}{$\begin{array}{l}\text { Origine } \\
\text { de la santé }\end{array}$} & Travail/occupation & 8 & 4 & 5 \\
\cline { 2 - 5 } & Hérédité & 3 & 5 & 5 \\
\hline $\begin{array}{l}\text { Définition } \\
\text { de la santé }\end{array}$ & $\begin{array}{l}\text { Capacités } \\
\text { fonctionnelles }\end{array}$ & 5 & 5 & 4 \\
\hline $\begin{array}{l}\text { Conduite } \\
\text { de la santé }\end{array}$ & Bouger & 10 & 10 & 7 \\
\hline \multicolumn{2}{|l|}{} & $\mathrm{N}=10$ & $\mathrm{~N}=10$ & $\mathrm{~N}=9$ \\
\hline
\end{tabular}


En ce qui concerne les données rapportées au tableau 3, elles permettent de constater que certaines idées au sujet de la maladie sont également partagées par une majorité de répondantes, quel que soit le milieu d'appartenance. Ces idées font référence, tout comme pour la santé, à des dimensions extérieures à soi, à des attitudes ainsi qu'à des comportements spécifiques. Ainsi, pour plus des deux tiers de l'échantillon (20 répondantes sur 29), la maladie est évoquée comme une réalité inhérente au processus de vieillissement et directement reliée à l'usure normale du corps : On peut pas vieillir sans avoir des bobos. De même, la majorité des participantes ont fait référence à la maladie comme provenant des mauvaises habitudes de vie, en l'associant à la mauvaise alimentation, d'une part, et à l'usage du tabac ou de l'alcool, d'autre part. Par contre, l'idée de la mauvaise alimentation comme étant à l'origine de la maladie, même si elle se retrouve dans les discours des participantes des trois groupes, est évoquée différemment par les unes et par les autres. Dans ce cas, les femmes issues de l'élite et celles issues du milieu populaire font référence à la mauvaise alimentation de façon beaucoup plus générale (manger trop riche) que les femmes issues de la classe moyenne, lesquelles ont développé davantage l'idée du déséquilibre alimentaire (alimentation trop riche en féculents et apport trop faible en vitamines).

Enfin, trois idées principales ont traversé la majorité des discours concernant les conduites de maladie. II s'agit de l'idée de la consommation des services médicaux, de celle relative à l'idée de se soigner soi-même et de la pratique de repos. En effet, 28 des 29 femmes rencontrées ont parlé d'aller chez le médecin. II s'agit, avec celle de l'activité physique, de l'idée la plus souvent partagée par les participantes, sans égard à leur milieu social d'appartenance. Fondamentalement, les répondantes associent l'utilisation des services médicaux à un privilège: Je me trouve vraiment chanceuse. Y'en a qui ont ben de la misère à trouver un médecin.

Par ailleurs, une forte proportion de répondantes ont fait état de différentes conduites de maladie caractérisées par l'idée de se soigner par soi-même. En effet, si la consultation médicale est mentionnée par la presque totalité des participantes, la majorité des femmes rencontrées font également référence à différentes 
activités ou à différents moyens personnels qu'elles adoptent comme pratiques de gestion de la maladie. Ainsi, 20 des 29 femmes interrogées ont fait état de différentes activités, que nous avons regroupées sous le thème des auto-pratiques de soins. Globalement, les répondantes ont mentionné une variété de pratiques qu'elles mettent en œuvre en réponse à une tout aussi grande variété de malaises.

\section{Tableau 3}

Les idées communes au sujet de la maladie parmi les répondantes des différentes classes sociales

\begin{tabular}{|l|l|c|c|c|}
\hline \multicolumn{2}{|c|}{ Catégorie } & Populaire & Moyenne & Élite \\
\hline \multirow{2}{*}{$\begin{array}{l}\text { Origine } \\
\text { de la maladie }\end{array}$} & Usure normale & 7 & 8 & 5 \\
\cline { 2 - 5 } & $\begin{array}{l}\text { Mauvaises } \\
\text { habitudes de vie }\end{array}$ & 8 & 7 & 8 \\
\hline \multirow{2}{*}{$\begin{array}{l}\text { Conduite } \\
\text { de la maladie }\end{array}$} & $\begin{array}{l}\text { Consommation } \\
\text { des soins médicaux }\end{array}$ & 9 & 10 & 9 \\
\cline { 2 - 5 } & $\begin{array}{l}\text { Auto-pratique } \\
\text { des soins }\end{array}$ & 8 & 5 & 7 \\
\cline { 2 - 5 } & $\begin{array}{l}\text { Cessation des } \\
\text { activités - Repos }\end{array}$ & 6 & 5 & 3 \\
\cline { 2 - 5 } & $\mathrm{N}=10$ & $\mathrm{~N}=10$ & $\mathrm{~N}=9$ \\
\hline
\end{tabular}

Une analyse plus fine des énoncés formant cette catégorie nous a toutefois permis de déceler des différences dans les moyens utilisés selon l'appartenance de milieu des répondantes, notamment à l'égard des ceux utilisés lors d'épisodes de rhume, de grippe et d'autres malaises considérés comme bénins ou, à tout le moins, ne justifiant pas une consultation médicale. Ainsi, huit des dix femmes du milieu populaire ont évoqué la consommation d'aspirine et de tylenol parmi les moyens qu'elles prennent pour contrer plusieurs malaises dont un épisode de grippe, les douleurs associées aux symptômes de l'arthrite, les douleurs musculaires générales, le mal de tête, etc. : Je prends des tylenols, des choses de même. Viennent ensuite la soupe, l'eau chaude, le jus, le camphre, mentionnés parmi les moyens qu'elles prennent pour se 
soigner. Ici, la pertinence du moyen choisi est liée avant tout au savoir d'expérience et un même moyen peut être utilisé pour plusieurs types de malaises. Cinq femmes de la classe moyenne ont également indiqué des moyens qu'elles prennent pour contrer des malaises. Par contre, à la différence du groupe précédent, un moyen précis est généralement associé à un symptôme ou à un malaise précis. L'aspirine pour éclaircir le sang, l'échinacée et la vitamine $\mathrm{C}$ pour un rhume, un sirop vendu sans ordonnance pour arrêter de tousser, la marche et la relaxation pour la douleur aux jambes, etc. Quant aux femmes appartenant à l'élite, elles ont fait référence de façon plus explicite à des pratiques alternatives de soins qui favorisant leur mieux-être (le yoga, le jeûne). Tout comme les femmes de milieu populaire, elles ont évoqué la responsabilité personnelle d'agir pour retrouver un état de bien-être : Il faut que tu te prennes en main. Ainsi, si une proportion importante des femmes rencontrées font état de différents soins qu'elles s'administrent elles-mêmes, une lecture plus approfondie de cette catégorie laisse voir les différences dans les moyens qu'elles utilisent et l'usage qu'elles en font.

\section{ANALYSE DES RÉSULTATS}

Nous avons vu précédemment que l'idée la plus souvent exprimée par les femmes ayant participé à cette étude renvoie à la consommation de services médicaux comme conduite de maladie. Or, cette évocation univoque du recours au médecin comme conduite de maladie chez les femmes interrogées rejoint directement l'analyse de plusieurs auteurs en sociologie de la santé au sujet de la place centrale du médecin dans l'institution médicale et de la signification accordée à l'institution sociale que représente le système de santé (Paquet, 1989; Adam et Herzlich, 1994; Massé, 1995).

D'autre part, les femmes évoquent majoritairement l'importance pour elles d'être actives, de s'occuper, comme conduite de santé. À leur avis, le fait de demeurer actif permet de conserver sa santé. En effet, la quasi-totalité des femmes rencontrées ont fait référence à l'activité physique comme moyen pour conserver sa santé, indiquant clairement un lien entre le maintien de leur santé et l'activité du corps. Par contre, les mêmes participantes évoquent 
l'inactivité ou la cessation d'activité comme conduite privilégiée de maladie. Or, on aurait pu s'attendre à ce qu'elles insistent au contraire sur l'importance de demeurer actif le plus possible, même en cas d'épisode de maladie. Nous avons rattaché ce résultat au fait que notre échantillon est composé pour la majeure partie de femmes membres de l'un ou l'autre club local de l'âge d'or. Or, ces associations locales proposent depuis quelques années un programme de conditionnement physique intitulé "Grouille ou rouille », offert dans la quasi-totalité des paroisses de la région urbaine du grand Moncton. Nous sommes d'avis que le discours des femmes rencontrées est influencé au moins en partie par l'expérience de participation à ce programme, rejoignant ainsi les propos de Delisle et Ouellet (2002) sur l'influence de la participation sociale sur les opinions, les attitudes et les comportements en matière de gestion de sa santé.

De même, la logique du repos comme une conduite de maladie a été le plus souvent présentée comme relevant d'un ordre naturel des choses, auquel on recourait autrefois de façon automatique : C'était le repos dans ce temps-là. Plus encore, dans certains cas, les participantes ont fait part de l'écart qu'elles perçoivent entre le mode de gestion institutionnel de la maladie d'aujourd'hui et le savoir d'expérience. Ainsi, la référence au travail comme étant à l'origine de la santé et la valeur accordée au fait d'être actif pour demeurer en santé co-existent avec l'idée du repos et de la cessation d'activités pour recouvrer la santé en période de maladie. La présence simultanée de ces idées que partagent une majorité de répondantes nous semble un élément important au regard de la compréhension des logiques d'articulation des représentations que se font les femmes âgées de la santé, de la maladie et de leur mode de gestion respectif.

En deuxième partie d'analyse, nous avons cherché à mettre en lumière les différences les plus significatives dans les idées exprimées par les femmes rencontrées selon leur milieu d'appartenance. À cet effet, nous avons retenu les idées qui, malgré leur possible présence dans l'un ou l'autre groupe de répondantes, étaient en majorité le fait de répondantes issues d'un milieu donné, contribuant ainsi à mettre en lumière les différences plus 
significatives au regard des milieux sociaux d'appartenance ${ }^{5}$. Nous avons retenu comme élément représentatif du discours des femmes issues d'un milieu donné les catégories où plus de $50 \%$ des énoncés avaient été émis par les participantes de ce milieu. Le prochain tableau rend ainsi compte des éléments les plus significatifs du discours des femmes issues du milieu populaire et qui se distinguent des éléments du discours qu'elles partagent avec les autres participantes.

Les données figurant au tableau 4 permettent de constater que l'idée de la santé correspondant à un état de fait auquel échappe l'emprise personnelle est largement plus présente chez les femmes du milieu populaire que dans les autres groupes. Ainsi, sur les huit participantes ayant fait référence à l'idée selon laquelle la santé vient du destin ou du Bon Dieu, cinq appartiennent au milieu populaire: C'est le Bon Dieu qui l'envoie, la santé. De même, contrairement aux femmes des autres groupes, sept femmes du milieu populaire ont défini la santé comme étant une richesse, un don de Dieu, une valeur inestimable, un trésor précieux offert. Une femme seulement dans chacun des deux autres groupes avait exprimé cette idée.

Nous sommes d'avis que cette idée de la santé définie comme un trésor précieux est en accord avec une conception de la santé venant d'au-delà de soi, ce qui contraste avec le discours des femmes des autres milieux où la référence à la volonté et à la responsabilité personnelles est beaucoup plus importante et qui rejoint les propos de plusieurs auteurs au sujet des rapports entre la culture populaire et la prévention de la maladie (Massé, 1995; Lacourse, 1998). De la même manière, comme l'envers du destin heureux, des douze femmes qui sont d'avis que la maladie est d'origine inconnue, huit appartiennent au milieu populaire: D'où ça vient ça la maladie? Je l'sais pas. On le sait pas. Enfin, la moitié des participantes issues du milieu populaire ont parlé de la maladie provoquée par de mauvaises conditions matérielles de vie, contre trois parmi les autres répondantes. Ma mère, elle a

5. De manière à mettre en lumière les éléments caractéristiques du discours des unes et des autres, nous avons éliminé de l'analyse comparative les idées plus marginales émises par moins de 8 répondantes sur 29, soit moins de $29 \%$ de l'échantillon total des participantes. 
attrapé la tuberculose, elle avait soigné sa sœur qui avait été travailler aux États-Unis dans une usine et ce n'était pas sanitaire dans ce temps-là.

\section{Tableau 4}

Santé et maladie : les thèmes du discours propres aux répondantes de la classe populaire

\begin{tabular}{|l|l|c|c|c|}
\hline \multicolumn{2}{|c|}{ Catégories } & Populaire & Moyenne & Élite \\
\hline $\begin{array}{l}\text { Origine } \\
\text { de la santé }\end{array}$ & $\begin{array}{l}\text { Destin, Bon Dieu, } \\
\text { chance }\end{array}$ & 5 & 0 & 3 \\
\hline $\begin{array}{l}\text { Définition } \\
\text { de la santé }\end{array}$ & $\begin{array}{l}\text { Don de Dieu, } \\
\text { trésor précieux }\end{array}$ & 7 & 1 & 1 \\
\hline \multirow{2}{*}{$\begin{array}{l}\text { Origines } \\
\text { de la maladie }\end{array}$} & Inconnue & 8 & 3 & 1 \\
\cline { 2 - 5 } & $\begin{array}{l}\text { Conditions } \\
\text { matérielles }\end{array}$ & 5 & 2 & 1 \\
\hline \multicolumn{2}{|l|}{} & $\mathrm{N}=10$ & $\mathrm{~N}=10$ & $\mathrm{~N}=9$ \\
\hline
\end{tabular}

En somme, au-delà des thèmes communs, le discours spécifique des participantes issues du milieu populaire laisse voir la part que prend l'impondérable, l'incontrôlable et l'inévitable dans les sens donnés à la santé et à la maladie et rejoint les propos de nombreux autres auteurs, dont Marie-Thérèse Lacourse selon qui cette part du destin ou de l'au-delà de soi est au fond révélatrice de l'expérience quotidienne, où, dans un contexte de multiples privations et contraintes, l'exercice de la volonté personnelle et du choix individuel occupe une place marginale (Lacourse, 1998).

Par ailleurs, comme l'illustre le tableau 5, davantage de femmes issues de la classe moyenne ont fait référence à la question de la force morale et de la volonté personnelle comme étant à l'origine de la santé, comparativement aux participantes des autres groupes où cette idée est évoquée dans une proportion beaucoup plus faible : Ben mentalement quand une personne a une attitude positive, je trouve que ça c'est un premier facteur, peu importe l'état de son physique. 
Plus encore, l'importance que les femmes issues de la classe moyenne accordent à l'attitude personnelle en ce qui concerne cette fois la gestion d'un épisode de maladie est encore plus grande que dans les autres groupes de participantes. En effet, parmi les neuf femmes ayant fait part d'une attitude positive comme un moyen favorisant le retour à la santé en période de maladie, six appartenaient à la classe moyenne. Moi je trouve que ça vient beaucoup de toi-même, c'est ta manière de voir la vie, c'est qu'est-ce que toi tu veux, c'est la manière que tu vois la vie. Tu fais des choses pour être en santé. Ici, la référence à la responsabilité personnelle est centrale. Enfin, le discours des femmes issues de la classe moyenne se distingue du discours des autres participantes par l'ampleur de la référence à la science comme contribuant à la santé des individus, rejoignant entre autres les propos de Gisèle Ampleman sur la valeur accordée à la connaissance intellectuelle des choses et des événements caractéristique de la culture bourgeoise, laquelle exerce par ailleurs une influence directe sur les attitudes des intervenantes œuvrant dans le champ de la santé (Ampleman, 1987). En effet, sur les dix femmes ayant fait mention de l'évolution des connaissances scientifiques comme contribuant directement à la santé des personnes, notamment par l'effet sur la longévité, six étaient de la classe moyenne : Les vieillards, ça vit très vieux, donc il y a sûrement une amélioration dans la santé qu'on n'avait pas auparavant, parce que la moyenne d'âge grandit avec les années, donc la santé s'améliore. En fait, pour les participantes, la science contribue à la santé de deux manières : parce qu'elle permet de combattre des maladies, donc d'augmenter la durée de vie, et aussi parce qu'elle engendre un savoir nouveau en matière de prévention des maladies et de maintien de la santé. En fait, dans nos foyers actuellement, ils sont suivis de très près dans la nourriture. Les activités, ils ont tout ce qu'il faut pour les garder alertes en santé. 


\section{Tableau 5}

\section{Santé et maladie : les thèmes du discours propres aux répondantes de la classe moyenne}

\begin{tabular}{|l|l|c|c|c|}
\hline \multicolumn{2}{|l|}{ Catégories } & Populaire & Moyenne & Élite \\
\hline \multirow{2}{*}{$\begin{array}{l}\text { Origine } \\
\text { de la santé }\end{array}$} & $\begin{array}{l}\text { Force morale, volonté } \\
\text { personnelle }\end{array}$ & 2 & 8 & 3 \\
\cline { 2 - 5 } & $\begin{array}{l}\text { Évolution des connais- } \\
\text { sances scientifiques }\end{array}$ & 3 & 6 & 1 \\
\hline $\begin{array}{l}\text { Conduite } \\
\text { de la } \\
\text { maladie }\end{array}$ & $\begin{array}{l}\text { S'occuper - s'activer, } \\
\text { s'impliquer dans la } \\
\text { gestion de sa maladie }\end{array}$ & 0 & 6 & 3 \\
\cline { 2 - 5 } & $\mathrm{N}=10$ & $\mathrm{~N}=10$ & $\mathrm{~N}=9$ \\
\hline
\end{tabular}

En somme, pour les femmes issues de la classe moyenne, la santé n'est pas un don du ciel. Elle se "gagne » par l'adoption de comportements et l'exercice de choix volontaires. Dès lors, qu'il passe par l'acquisition du savoir ou par l'attitude personnelle face à la santé/maladie, le discours des femmes issues de la classe moyenne se démarque de celui des femmes du milieu populaire par l'accent mis sur le choix et la responsabilité individuelle, ce qui témoigne à notre avis de l'emprise que ces femmes exercent sur leur environnement.

De manière générale, le discours des femmes composant l'élite se distingue moins du discours des autres groupes. Concrètement, les opinions de ces femmes en ce qui regarde la santé et la maladie rejoignent tantôt les propos des participantes issues du milieu populaire, tantôt ceux des participantes appartenant à la classe moyenne. En somme, si nous avons pu dégager des éléments distinctifs du discours dans les deux autres groupes, ici seule l'évocation du mode de vie moderne comme étant associé aux origines de la maladie se distingue comme un élément caractéristique du discours des femmes issues de l'élite locale. Les énoncés de cette catégorie font référence plus particulièrement au rythme de la vie moderne et à son effet sur la santé des personnes. On court tout le temps aujourd'hui. C'est vrai, on vit plus vieux que nos ancêtres, mais c'étaient pas des gens stressés, ces 
gens-là. [...] je crois que ça aide pas parce qu'on a tout le temps des gens qui ont des problèmes de santé et qui sont jeunes et ils ont déjà des problèmes de santé.

\section{Tableau 6}

\section{Santé et maladie : les thèmes du discours propres aux répondantes de la classe élite}

\begin{tabular}{|c|c|c|c|c|}
\hline \multicolumn{2}{|c|}{ Catégories } & Populaire & Moyenne & Élite \\
\hline $\begin{array}{l}\text { Origine de } \\
\text { la maladie }\end{array}$ & $\begin{array}{l}\text { Mode de vie } \\
\text { moderne }\end{array}$ & 2 & 3 & 5 \\
\hline & & $N=10$ & $\mathrm{~N}=10$ & $\mathrm{~N}=9$ \\
\hline
\end{tabular}

Par ailleurs, cette évocation d'un contraste entre le monde ancien et le monde actuel traverse à plusieurs occasions le discours des femmes de l'élite sur la santé et la maladie, le monde du passé étant relié à la santé et celui d'aujourd'hui, avec les changements qu'il a apportés au mode de vie des personnes, étant davantage porteur de maladie. Nous avons en effet retrouvé cette idée du contraste entre l'ancien et le moderne dans les propos sur l'occupation et le travail comme étant des facteurs de la santé.

Nous autres quand on était à l'école, on nous a montré à tricoter, on nous a montré à crocheter, beaucoup de choses, pis moi j'ai continué. Tout le temps, tout le temps. Pis je crois que j'ai jamais pris de pilules pour les nerfs. [...] travailler, travailler physiquement c'est très bon, puis aujourd'hui hein, y'a beaucoup moins de travail physique.

D'autres encore ont fait le lien entre le passé et le présent à travers le discours sur l'usage de l'alcool ou du tabac, jugé moins nocif hier qu'aujourd'hui. Cette évocation d'une distance entre l'ordre des choses du monde passé et celui du monde actuel nous semble également significative de la position de classe des femmes de ce groupe et, partant, de leur rapport au monde. Concrètement, la majorité des femmes de ce groupe viennent du milieu populaire ou de la petite-bourgeoisie artisanale. Leur ascension quant à leur position de classe participe du mouvement d'essor de la communauté acadienne qui a pris naissance dans les années 
1960 avec entre autres l'arrivée d'un premier ministre francophone à la tête du gouvernement provincial. Les femmes âgées de notre recherche comptent donc parmi les premières générations de femmes composant l'élite locale acadienne dans la région de Moncton. Par conséquent, nous croyons que nos résultats auraient pu être différents si la recherche avait été conduite auprès de femmes âgées anglophones issues du même milieu social.

\section{CONCLUSION}

L'analyse catégorielle que nous avons faite des entretiens montre avec suffisamment d'éloquence que les représentations de la santé et de la maladie des femmes de milieu populaire et celles issues de la classe moyenne, même si elles présentent certains éléments de ressemblance, diffèrent largement du point de vue de l'appartenance à un milieu donné. Toutefois, l'analyse des entretiens menés auprès des femmes appartenant à l'élite ne nous permet pas de dégager une représentation de la santé qui se distingue des deux autres milieux sociaux pris en compte dans cette étude. En effet, les femmes de l'élite que nous avons rencontrées considèrent, tout comme les femmes du milieu populaire, la maladie et la santé comme des réalités déterminées au-delà de soi, sur lesquelles elles ont peu ou pas de prise. En même temps, elles insistent sur l'importance de la force morale et de la responsabilité personnelle, rejoignant par là les propos caractéristiques des femmes issues de la classe moyenne. Fondamentalement, notre recherche s'appuyait sur une conception de la vieillesse comme étant déterminée par la position occupée dans la structure des rapports sociaux durant la période de la vie dite active. Tout comme Anne-Marie Guillemard, nous avons postulé au départ qu'il existe « des vieillissements et des vieillesses en fonction des positions de classe dans une même société 》(Guillemard, 1993, p. 7). Les résultats obtenus dans notre étude rejoignent à cet égard les propos de Guillemard et de plusieurs autres auteurs au sujet de l'influence des rapports de production sur les manières de voir, de connaître et d'agir ou, plus largement, sur la culture (Lalive D'Épinay, 1991; Dumont, 1994). 
Ces résultats invitent également à tenir compte davantage de cette culture de milieu dans la pratique sociosanitaire auprès des personnes âgées. À titre d'exemple, on peut penser que les propos contenus dans le dernier bulletin Réflexions, destiné aux personnes âgées et publié par le ministère des Services familiaux et communautaires du Nouveau-Brunswick, rejoignent aisément les personnes âgées issues de la classe moyenne. Ils rejoignent cependant peut-être beaucoup moins les personnes de milieu populaire, en présentant une conception du vieillissement en santé comme le résultat d'une prise en main individuelle.

\begin{abstract}
Chaque jour, sans exception, nous pouvons contrôler divers facteurs qui influent sur notre processus de vieillissement [...] Nous avons bien des choix à faire autant à notre mode de vie [...] Nous exerçons parfois plus de contrôle que nous le réalisons. En réalité, nous contrôlons tout ce qui peut affecter la façon dont nous vieillissons. Que chacun de nous s'efforce de prendre les décisions qui lui permettront de rester en santé et de vivre plus longtemps (Services familiaux et communautaires, 2003).
\end{abstract}

Chez les femmes issues de la classe moyenne, nous l'avons vu, la santé n'est pas d'abord un don, elle est méritée. Cependant, comment ce discours s'arrime-t-il avec l'expérience des femmes issues du milieu populaire, pour lesquelles santé et maladie s'expliquent surtout en référence au travail, au destin, à la chance/malchance, à Dieu, à une hérédité malencontreuse ou à l'inconnu? Ici, le défi semble être celui de l'arrimage avec les valeurs propres aux personnes de milieux populaires. C'est là que réside à notre avis le rôle spécifique du service social dans le champ de la promotion de la santé, comme traducteur des références culturelles pour des pratiques qui tiennent compte davantage de la réalité telle que vécue et des problèmes tels que ressentis par les personnes de milieu populaire. Dans cette optique, l'engagement récent de l'Association canadienne des travailleurs sociaux (ACTS) au sein de la Coalition canadienne des professions de la santé pour des pratiques préventives nous semble prometteuse. La coalition, qui regroupe neuf associations nationales dans le champ de la santé, s'est donné comme objectif : 
[...] d'aider les professionnels à acquérir les connaissances et les habiletés nécessaires pour améliorer les composantes de leurs pratiques qui touchent la prévention et la promotion. [...] chaque membre de la Coalition partage ses habiletés et son expérience afin d'améliorer la santé de la collectivité, ceci au-delà de ce qu'un groupe ou une discipline pourrait envisager ou accomplir à lui seul (Association canadienne des travailleuses et travailleurs sociaux, 2003).

De même, la tenue à Québec, en mai 2004, de la quatrième Conférence internationale du service social en santé et en santé mentale témoigne de l'importance pour les travailleurs sociaux et les travailleuses sociales de témoigner du fait que l'état de santé des personnes est d'abord le résultat de l'interaction entre les individus et leur milieu de vie. Au moment où un large consensus existe autour de la notion de la santé comme résultant de l'interaction de plusieurs facteurs ou déterminants, le service social est tout désigné pour faire valoir la dimension culturelle du processus de gestion de la santé des individus et sa nécessaire prise en compte par le système de soins. 


\section{RÉFÉRENCES BIBLIOGRAPHIQUES}

Adam, P. et C. HeRzlich (1994). Sociologie de la maladie et de la médecine, Paris, Nathan.

AlLAIN, G. (1998). "L'entrepreneurship minoritaire et le développement économique local : le rôle des gens d'affaires dans la croissance récente du grand Moncton ", Communication, Congrès de l'ACFAS, Université Laval, Sainte-Foy, mai 1998.

AMPLEMAN, G. (1987). "La formation des intervenantes en santé communautaire en milieux populaires » dans G. Ampleman, J. Barnabé, Y. Comeau, G. Doré, R. Duhaime, L. Gaudreau, C. Humbert, J. Lacroix, L. Lebœuf et M. Matte, Pratiques de conscientisation II, Québec, Collectif québécois d'édition populaire.

ANKRI, J. et J.-C. HenRARD (1994). «Perceptions de la santé chez les personnes âgées », Gérontologie et Société, n 71, p. 101-106.

ARCURY, T.A., S.A. QUANDT et R.A. BELL (2001). " Staying healthy : The salience and meaning of health maintenance behaviors among rural older adults in North Carolina», Social Science and Medicine, $\mathrm{n}^{\circ} 53$, p. 1541-1556.

ASSOCIATION CANADIENNE DES TRAVAILLEUSES ET TRAVAILLEURS SOCIAUX (ACTS). La coalition des professions de la santé pour des pratiques préventives [http://CASW-ACTS/advocacy/coalitions_f.htm].

Page consultée le 7 juillet 2003.

BARITEAU, C. (1985). "Crise et stratégies locales: Plessisville, SaintGeorges, Thetford Mines ", Anthropologie et sociétés, vol. 9, $\mathrm{n}^{\circ} 2$, p. 57-84.

CALNAN, M. (1986). "Maintaining health and preventing illness : A comparison of the perceptions of women from different social classes ", Health Promotion, vol. 1, $n^{\circ}$ 2, p. 167-177.

Colin, C., F. Ouellet, G. Boyer et C. Martin (1992). Extrême pauvreté, maternité et santé, Montréal, Saint-Martin. 
Corin, E., J. Tremblay, T. Sherif et L. Bergeron (1984). «Entre les services professionnels et les réseaux sociaux: les stratégies d'existence des personnes âgées ", Sociologie et sociétés, vol. XVI, $\mathrm{n}^{\circ} 2$, p. 89-104.

DAIGLE, J. (dir.) (1993). L'Acadie des Maritimes, Chaire d'études acadiennes, Université de Moncton.

Dale, A., G.N. GILBERT et S. ARBeR (1985). «Integrating women into class theory ", Sociology, vol. 19, n 3 , p. 384-408.

DAUNAIS, J.-P. (1992). «L'entretien non directif », dans B. Gauthier (dir.), Recherche sociale. De la problématique à la collecte des données, $2^{\mathrm{e}}$ éd., Sainte-Foy, Presses de l'Université du Québec, p. 273-293.

DE KONINCK, M. et L. GUYON (1985). " Le système de santé et sa clientèle ", dans J. Dufresne et al., Traité d'anthropologie médicale, Institut québécois de recherche sur la culture, Sainte-Foy, Presses de I'Université du Québec, p. 537-564.

DelisLe, M.-A. et H. OUELLET (2002). Les « vieux copains » et leur santé : participation sociale, entraide et recours aux services chez les aînés, Québec, Les Presses de l'Université Laval.

DORÉ, G. (1988). Mouvement populaire et politique partisane au Québec (MPPPQ). Dialectique de l'aliénation et de l'identité dans les élaborations symboliques d'un conflit latent. Description détaillée du projet. Projet de recherche, École de service social, Université Laval, texte inédit.

DORÉ, G. (1991). «L'organisation communautaire et l'exclusion politique des classes populaires », Service social, vol. 40, n 3, p. 82-103.

Dumont, F. (1994). Le lieu de l'homme, Montréal, Bibliothèque québécoise.

FerRini, R., S. Edelstein et E. BARRET-ConNor (1994). "The association between health beliefs and health behavior change in older adults ", Preventive Medicine, vol. 23, p. 1-5.

GerRitsen, J.C., E.W. WolfFensperger et W.J.A. VAN DEN Heuvel (1990). "Rural-urban differences in the utilization of care by the elderly », Journal of Cross-Cultural Gerontology, vol. 5, p. 131-147. 
GoDIN, I. (1993). "La culture des familles et leur santé / Family Culture and Family Health», Arch Public Health, n ${ }^{\circ}$ 51, p. 89-96.

GRZYWACZ, J.G. (2000). "Socioeconomic status and health behaviors among Californians ", Health, IIIness and Use of Care : The Impact of Social Factors, vol. 18, p. 121-149.

GUILLEMARD, A.-M. (1993). "Le vieillissement, un processus socialement défini », Le Gérontophile, vol. 15, n 4, p. 7-12.

JODELET, D. (1992). "Les représentations sociales de la maladie mentale dans un milieu rural français : genèse, structure, fonction ", dans U. Flick (dir.) (1992). La perception quotidienne de la santé et de la maladie, Paris, L'Harmattan. p. 321-346.

JOFFE, H. (1999). « Représentations sociales et psychologie de la santé », Pratiques psychologiques, vol. 4, p. 15-29.

KROUT, J.A. (1983). "Knowledge and use of services by the elderly : A critical review of the literature», International Journal of Aging and Human Development, vol. 17, $n^{\circ}$ 3, p. 153-167.

LaLIVe D'ÉPINAY, C. (1991). Vieillir ou la vie à inventer, Paris, L'Harmattan.

LEIGH, P.J. et J. FRIES (1993). « Associations among healthy habits, age, gender, and education in a sample of retirees", International Journal of Aging and Human Development, vol. 36, n 2, p. 139-155.

LUBben, J.E., P. WeILER et I. CHI (1989). « Gender and ethnic differences in the health practices of the elderly poor », Journal of Clinical Epidemiology, vol. 42, $\mathrm{n}^{\circ} 8$, p. 725-733.

MacFarlane, A. (1980). "Official statistics and women's health and illness », EOC Research Bulletin, Equal Opportunities Commission, $n^{\circ} 4$, p. $45-47$.

MASSÉ, R. (1995). Culture et santé publique, Montréal, Gaëtan Morin.

MITCHELL, J. et J.A. KROUT (1998). «Discretion and service use among older adults: The behavioral model revisited », The Gerontologist, vol. $38, n^{\circ} 2$, p. 159-168.

MOEN, E. (1978) "The reluctance of the elderly to accept help », Social Problems, vol. 25, n 3 , p. 293-303. 
ORGANISATION INTERNATIONALE DU TRAVAIL (2003). Classification internationale type des professions (CITP-88)

[http//www.ilo.org/public/french/bureau/stat/class/isco.htm].

PAQUeT, G. (1989). Santé et inégalités sociales. Un problème de distance culturelle, Québec, Institut québécois de recherche sur la culture.

ProvenCHeR, Y. (1999). "Les aînés de la MRC Denis-Riverin ou quand la recherche se met au service du changement ", Revue canadienne de service social, vol. $16, n^{\circ} 2$, p. 233-249.

ProvencheR, Y. (2002). "Personnes âgées de milieux populaires et professionnelles des services. Des représentations différentes de la santé et de la maladie ", Revue canadienne de service social, vol. $19, n^{\circ} 1$, p. 43-64.

Provencher, Y. et A. Lepage (2003). Classes sociales et représentations de la santé chez les femmes âgées francophones de la région du grand Moncton, Rapport final, École de travail social, Université de Moncton.

Roos, N.P. et C.A. MustaRD (1997). "Variation in health and health care use by socioeconomic status in Winnipeg, Canada: Does the system work well? Yes and no ", Milbank Quarterly, vol. 75, $\mathrm{n}^{\circ} 1$, p. 89-111.

SCHULZE, C. et L. WELTERS (1994). "L'incidence de l'âge et du sexe sur la conception de la santé ", dans U. Flick (dir.), La perception quotidienne de la santé et de la maladie: Théories subjectives et représentations sociales, Paris, L'Harmattan, p. 85-107.

SÉCA, J.-M. (2001). Les représentations sociales, Paris, Armand Colin/ VUEF.

SERVICES FAMILIAUX ET COMMUNAUTAIRES. "Réflexions - Conseils de santé pour les aînés ", dans Gouvernement du NouveauBrunswick, ministère des Services familiaux et communautaires, [http://www.gnb.ca/0017/seniors/Reflections/aging-f.asp].

Page consultée le 7 juillet 2003. 
SÉVIGNY, A. et A. VÉZINA (1996). "Les raisons qui motivent les aînés à utiliser ou à ne pas utiliser les services des organismes communautaires dans leurs stratégies de soutien à domicile ", Intervention, $n^{\circ} 103$, p. 26-35.

StATISTIQUE CANADA (2003). Concepts et variables

[http://www.statcan.ca/français/concepts/definitions/retirement_f.htm].

WILLIAMS, S.J. (1995). "Theorising class, health and lifestyles: Can Bourdieu help us? ", Sociology of Health and IIIness, vol. 17, $n^{\circ} 5$, p. 577-604. 